\section{Early rule-out pathways for myocardial infarction: is observational data enough?}

\author{
Kuan Ken Lee (i) , ${ }^{1}$ Nicholas L Mills (i) 1,2
}

Symptoms suggestive of an acute coronary syndrome remain one of the most common reasons for emergency department presentations worldwide. ${ }^{1}$ However, only a small proportion of patients have myocardial infarction, and therefore strategies to expedite evaluation are valuable both for patients and healthcare systems. ${ }^{2}$

The introduction of high-sensitivity cardiac troponin assays that are able to measure very low concentrations of cardiac troponin at high-precision has been one of the most important innovations in cardiovascular diagnostics in recent years. ${ }^{3}$ There is now substantial evidence demonstrating the excellent prognostic value of very low troponin concentrations, which have been incorporated into multiple early rule-out pathways for myocardial infarction. ${ }^{4}$ While some of these pathways are now recommended by national and international guidelines and have been implemented in clinical care, their performance has largely been evaluated in observational studies, and the true safety and efficacy of implementing these approaches is not known. ${ }^{5}$ Many of the observational studies were performed in small, selected patient populations and based on a retrospective analysis where the pathways were not used to guide clinical care. ${ }^{4}$ Conversely, there have been very few randomised controlled trials evaluating the safety and efficacy of these approaches. ${ }^{36}$

It is in this context that the LoDED (Limit of Detection and ECG Discharge) investigators must be congratulated for successfully delivering a prospective multicentre randomised controlled trial to evaluate the effectiveness of implementing an early rule-out pathway in adults with suspected cardiac chest pain and no evidence of myocardial ischaemia on the ECG. ${ }^{7}$ The LoDED strategy identified patients as eligible for discharge from the emergency department where

${ }^{1}$ BHF Centre for Cardiovascular Science, The University of Edinburgh, Edinburgh, United Kingdom

${ }^{2}$ Usher Institute, The University of Edinburgh, Edinburgh, United Kingdom

Correspondence to Professor Nicholas L Mills, BHF Centre for Cardiovascular Science, The University of Edinburgh, Edinburgh EH16 4SB, UK;

nick.mills@ed.ac.uk the troponin concentration at presentation was below a high-sensitivity cardiac troponin I or $\mathrm{T}$ assays limit of detection. They recruited a total of 629 patients across eight hospitals in England and Wales and randomised these patients 1:1 to either the early rule-out strategy or usual care using an open-label, blinded endpoint, parallel group design. In this trial, all sites used high-sensitivity cardiac troponin assays in usual care (five sites used a troponin $\mathrm{T}$ assay and three used a troponin I assay). However, it is important to note that in half the sites, the limit of detection was already in use as part of the usual care pathway in combination with a validated risk stratification score. The primary outcome was discharge from hospital within 4 hours of arrival without a major adverse cardiac event (MACE), which was defined as cardiac death, type 1 myocardial infarction or emergency coronary revascularisation within 30 days. All MACE outcomes were adjudicated by a panel blinded to the study group and presentation troponin concentrations. The 4-hour timepoint was prespecified because this is an important operational standard for emergency departments within the National Health Service in the UK.

The LoDED trial reported that a larger proportion of patients were safely discharged within 4 hours in the group randomised to the LoDED strategy compared with usual care (46\% vs $37 \%$, respectively) although this was not significantly different (pooled adjusted OR, 1.58 (95\% CI 0.84 to 2.98$)$ ). There was also no difference in the rates of MACE at 30 days in patients randomised to the LoDED strategy compared with usual care $(8 \%$ vs 5\% respectively, OR 1.50 95\% CI 0.76 to 3.02$)$ ), median time to discharge (4.4 (3.2-6.8) hours vs $5.0(3.4-7.4))$, patient satisfaction $(51(44-55)$ vs $50(44-55))$ or secondary care costs up to 30 days post discharge ( $£ 429$ ( $£ 302$ to $£ 651$ ) vs $£ 527$ (£391 to $£ 720))$.

There are a number of potential reasons why the trial did not demonstrate a meaningful difference in efficacy between the LoDED strategy and usual care. First, half the participating hospital sites already used the limit of detection threshold as part of the usual care early rule-out pathway, which attenuated the effectiveness of the intervention. Indeed, there was significant heterogeneity across the eight hospital sites for the primary outcome $\left(\mathrm{I}^{2}=61 \%, \mathrm{p}=0.01\right)$. Interestingly, in a subgroup analysis, there was a significant improvement in efficacy in sites that did not already use the limit of detection in usual care (OR 2.87 (1.077.69)), but there was no difference in sites that did use this threshold (OR 0.93 (0.54-1.59)). However, as the authors acknowledged, this is not the only explanation for the overall result, because only two out of the four sites that did not use the limit of detection in their usual care improved. Other factors such as differences in intensity of each department, patient population and other local policies might have influenced length of stay across hospital sites. Second, it is possible that the trial was underpowered to observe a significant difference between the rule-out strategies. The trial was powered to detect a $9 \%$ difference in the primary outcome based on an anticipated primary outcome rate of $17 \%$ in the LoDED arm vs $9 \%$ in the usual care arm. In fact, the trial observed a much higher rate of early discharge within 4 hours in both arms $46 \%$ in those randomised to the LoDED strategy vs $37 \%$ in those randomised to standard care). This meant that the trial would have required approximately twice the sample size of over 1200 patients to detect a $9 \%$ difference in efficacy with $90 \%$ power and two-sided alpha of 0.05 . Third, the trial population was relatively young (mean age of $53.8 \pm 16.1$ years) and low-risk (prior history of coronary artery disease $(13 \%)$, diabetes mellitus $(10 \%)$, hypertension (27\%), hyperlipidaemia $(15 \%))$ in comparison with other studies of unselected patients presenting to the emergency department with suspected acute coronary syndrome. ${ }^{3}$ It is possible that the recruitment process has introduced a degree of selection bias. These low-risk patients may have presented with lower diagnostic complexity, which may have limited the added value of the LoDED strategy. Finally, while the rationale for using the limit of detection is well supported by evidence from observational studies and recommended by international guidelines, recent evidence suggests that higher risk stratification thresholds of cardiac troponin may have greater efficacy with similar safety. ${ }^{8}$

Although there may be some uncertainty in the interpretation of the 
LoDED trial, it does provide important insights into the use of high-sensitivity troponin in the risk stratification of patients with suspected acute coronary syndrome. A high degree of adherence to the new pathway $(88 \% \quad(95 \%$ CI $81 \%$ to $93 \%$ )) was observed, which likely reflects ease of applicability of the LoDED pathway and a high-level of confidence in the prognostic value of high-sensitivity cardiac troponin among clinicians. It is important to note that although patients were randomised to two different pathways, clinical decisions were made entirely at the discretion of the treating clinician. Furthermore, regardless of pathway allocation, no patients discharged with a troponin concentration below the limit of detection had a MACE outcome within 30 days. Although this study was not powered to evaluate safety, this further corroborates findings from observational studies on the excellent prognostic value of very low cardiac troponin concentrations. Finally, LoDED included only those who presented within 6 hours of symptom onset and therefore enriched for patients who presented early following the onset of chest pain, with a median of time from symptom onset to arrival of 2.3 hours (IQR, 1.5-3.6). A lack of evidence of harm is reassuring, but will need to be further investigated in future clinical trials enrolling a broader range of patients before recommending this approach for patients who present within 3 hours of symptom onset.

In summary, the LoDED trial did not demonstrate that using a high-sensitivity cardiac troponin concentration below the limit of detection at presentation was more effective than usual care to rule-out myocardial infarction in the emergency department. Nevertheless, there were a number of encouraging insights which require further investigation. The LoDED trial and other randomised controlled trials addressing this topic ${ }^{6}$ are much needed to raise the quality of evidence used to inform national and international clinical practice guidelines. These pathways are used to guide some of the most important decisions in practice, such as hospital admission for inpatient care, referral for specialist evaluation, selection for further diagnostic testing and prescription of disease modifying therapies, which have profound implications on individual patient care and use of scarce hospital resources. Clinicians and healthcare systems must therefore demand nothing short of the highest quality of evidence from randomised trials to inform the implementation of these strategies.

Contributors KKL and NLM wrote the editorial together.

Funding KL and NLM are supported by a Clinical Research Training Fellowship (FS/18/25/33454), and the Butler Senior Clinical Research Fellowship (FS/16/14/32023) and a Research Excellence Award (RE/18/5/34216), respectively, from the British Heart Foundation.

Competing interests NLM has received honoraria or consultancy from Abbott Diagnostics, Roche Diagnostics, Siemens Healthineers and LumiraDx. KKL has received honoraria from Abbott Diagnostics.

Patient and public involvement Patients and/or the public were not involved in the design, or conduct, or reporting, or dissemination plans of this research.

Patient consent for publication Not required.

Provenance and peer review Commissioned; internally peer reviewed.

\section{(2) OPEN ACCESS}

Open access This is an open access article distributed in accordance with the Creative Commons Attribution Non Commercial (CC BY-NC 4.0) license, which permits others to distribute, remix, adapt, build upon this work non-commercially, and license their derivative works on different terms, provided the original work is properly cited, appropriate credit is given, any changes made indicated, and the use is non-commercial. See: http://creativecommons.org/ licenses/by-nc/4.0/.

(C) Author(s) (or their employer(s)) 2020. Re-use permitted under CC BY-NC. No commercial re-use. See rights and permissions. Published by BMJ.

Check for updates

To cite Lee KK, Mills NL. Heart 2020;106:1545-1546.

Published Online First 3 August 2020

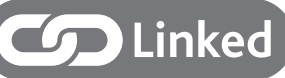

http://dx.doi.org/10.1136/heartjnl-2020-316692

Heart 2020;106:1545-1546.

doi:10.1136/heartjnl-2020-317012

ORCID iDs

Kuan Ken Lee http://orcid.org/0000-0003-3404-4098 Nicholas L Mills http://orcid.org/0000-0003-0533-7991

\section{REFERENCES}

1 Stepinska J, Lettino M, Ahrens I, et al. Diagnosis and risk stratification of chest pain patients in the emergency department: focus on acute coronary syndromes. A position paper of the acute cardiovascular care association. Eur Heart J Acute Cardiovasc Care 2020;9:76-89.

2 Anderson JL, Morrow DA. Acute myocardial infarction. N Engl J Med 2017:376:2053-64.

3 Shah ASV, Anand A, Strachan FE, et al. High-Sensitivity troponin in the evaluation of patients with suspected acute coronary syndrome: a stepped-wedge, clusterrandomised controlled trial. Lancet 2018;392:919-28.

4 Chapman AR, Anand A, Boeddinghaus J, et al. Comparison of the efficacy and safety of early rule-out pathways for acute myocardial infarction. Circulation 2017;135:1586-96.

5 Roffi M, Patrono C, Collet J-P, et al. 2015 ESC guidelines for the management of acute coronary syndromes in patients presenting without persistent ST-segment elevation. Eur Heart J 2016;37:267-315.

6 Chew DP, Lambrakis K, Blyth A, et al. A randomized trial of a 1-hour troponin T protocol in suspected acute coronary syndromes: the rapid assessment of possible acute coronary syndrome in the emergency department with high-sensitivity troponin T study (RAPID-TnT). Circulation 2019;140:1543-56.

7 Carlton EW, Ingram J, Taylor H, et al. Limit of detection of troponin discharge strategy versus usual care: randomised controlled trial. Heart 2020;106:1586-94.

8 Bularga A, Lee KK, Stewart S, et al. High-Sensitivity troponin and the application of risk stratification thresholds in patients with suspected acute coronary syndrome. Circulation 2019;140:1557-68. 